OPEN ACCESS

Edited by:

Hongbin Liu,

King's College London,

United Kingdom

Reviewed by:

Min Li,

Xi'an Jiaotong University, China

Riccardo Muradore,

University of Verona, Italy

*Correspondence:

Andualem Tadesse Maereg

maerega@hope.ac.uk

Specialty section:

This article was submitted to

Biomedical Robotics,

a section of the journal

Frontiers in Robotics and Al

Received: 09 May 2017

Accepted: 16 August 2017

Published: 06 September 2017

Citation:

Maereg AT, Nagar A, Reid D and

Secco EL (2017) Wearable

Vibrotactile Haptic Device for

Stiffness Discrimination during Virtual

Interactions.

Front. Robot. Al 4:42.

doi: 10.3389/frobt.2017.00042

\section{Wearable Vibrotactile Haptic Device for Stiffness Discrimination during Virtual Interactions}

\author{
Andualem Tadesse Maereg*, Atulya Nagar, David Reid and Emanuele L. Secco \\ Robotics Lab, Department of Mathematics and Computer Science, Liverpool Hope University, Liverpool, United Kingdom
}

In this paper, we discuss the development of cost effective, wireless, and wearable vibrotactile haptic device for stiffness perception during an interaction with virtual objects. Our experimental setup consists of haptic device with five vibrotactile actuators, virtual reality environment tailored in Unity 3D integrating the Oculus Rift Head Mounted Display (HMD) and the Leap Motion controller. The virtual environment is able to capture touch inputs from users. Interaction forces are then rendered at $500 \mathrm{~Hz}$ and fed back to the wearable setup stimulating fingertips with ERM vibrotactile actuators. Amplitude and frequency of vibrations are modulated proportionally to the interaction force to simulate the stiffness of a virtual object. A quantitative and qualitative study is done to compare the discrimination of stiffness on virtual linear spring in three sensory modalities: visual only feedback, tactile only feedback, and their combination. A common psychophysics method called the Two Alternative Forced Choice (2AFC) approach is used for quantitative analysis using Just Noticeable Difference (JND) and Weber Fractions (WF). According to the psychometric experiment result, average Weber fraction values of 0.39 for visual only feedback was improved to 0.25 by adding the tactile feedback.

Keywords: haptics, vibrotactile, virtual interactions, stiffness, psychophysics

\section{INTRODUCTION}

Our sense of touch is a combination of various different receptors (Rose and Mountcastle, 1959). It can tell texture, geometry, hardness, temperature, and weight. These sensory abilities will help us differentiate objects among others. Since sense of touch is a fusion of all these sensations, it makes it hard to achieve a full touch simulation using haptic devices (Díaz et al., 2006).

The haptic interaction of humans with objects and the environment is achieved by our fingeredhand. The human haptic perception synergically involves of two parts, which are kinesthetic haptic feedback and cutaneous haptic feedback (Jang and Lee, 2014). Cutaneous haptic perception is mediated by the responses of low threshold mechanoreceptors under the fingerpad skin within the contact area. The other is kinesthetic haptics referring to the sense of position and motion of the hand with the associated forces, mediated by the mechanoreceptors around the joints, tendons, and muscles.

Many commercial haptic devices are developed to provide kinesthetic haptic feedback. Some of these are Geomagic's Phantom, Force Dimension's Omega and Virtuose from Haption. However, these kinesthetic haptic devices are bulky and expensive, which reduces their impact on our daily life. On the other hand, finger tip cutaneous haptic devices can be low cost and portable solutions, even though, there can be some limitation in producing believable haptic sensations due to its lacking kinesthetic feedback (Minamizawa et al., 2010; Pacchierotti et al., 2012). Most haptic devices apply force and pressure by using electrical actuators, pneumatics, hydraulics, or other electromechanical 
systems (Connelly et al., 2010; Li et al., 2014). Devices such as data gloves use air bladders to harden and restrict you grip, so that you can feel objects in virtual reality. Wearable exoskeleton such as Cyber Grasp uses tendons and actuators to apply resistance to each fingers (Yang et al., 2008).

Vibrotactile displays have been developed for a variety of applications, including improving motor learning by enhancing the auditory and visual feedback (Lieberman and Breazeal, 2007), sensory substitution (Kaczmarek et al., 1991), and virtual social interaction and rehabilitation as well as tele-manipulation tasks (Van Erp, 2002; Galambos, 2012). Vibrotactile feedback can also significantly enhance touch perception for virtual gaming and prototyping application. Combining touch and vision allows the simultaneous extraction of perceptive process invariants, which is crucial for establishing the reciprocal connections that allow for higher order perception and categorization of objects and environments (Pabon et al., 2007).

The development in Virtual Reality (VR) technology is accelerating to make virtual experiences more immersive by providing sensory information that lets people feel as if they are inside the virtual environment. The sensory information can be visual, auditory, and tactile. To achieve a real time interaction, the VR system must be able to receive inputs from the real world and send feedback continuously and naturally (Carr and England, 1995).

In this paper, we explore the use of vibrotactile cutaneous feedback integrated with visual feedback of a virtual hand to replace the missing kinesthetic haptic feedback. Recent developments of haptic motor drivers, such as DRV2605 (from Texas instruments), reduces the limitation of open loop control of vibortactile motors. Such kind of drivers reduces the start up and break time of the vibration motors with closed loop feedback control algorithms and also enables us to trigger different types of waveforms already stored in their internal ROM. The DRV2605 also helps in reducing the rapid change in vibration strength using a digital level shifter integrated with PWM inputs, thereby reducing the annoying effect of vibration. The digital level shifter adjust the voltage levels to a fixed value as long as the input voltage meets the higher input voltage (VIH) and lower input voltage (VIL) levels. Even though such kind of drivers have been used in Mobile phone, Tablets, Remote controls, and Touch-enabled devices, their use in creating reliable control of cutaneous VR devices has not been explored. Here, we mainly focused on use of PWM modulated vibrotactile cues in order to increase immersion of the user in the virtual environment. This vibration modulation are done on amplitude and frequency of the signal based on the impact force during interaction with virtual objects. Sense of mechanical vibration of the skin can go as high as $10 \mathrm{kHz}$, but the ability to discriminate one vibration frequency from one another declines above $320 \mathrm{~Hz}$. In general, human can discriminate vibration cues in the range of frequency $50-400 \mathrm{~Hz}$ (Vicentini and Botturi, 2010).

The rest of the paper is organized as follows, in the section 2 a theoretical observation from previous literature's about the stiffness discrimination and the main objective of this paper is discussed then the apparatus, experimental setup and psychometric experiment are described in section 3. In section 4 the experimental results are reported in the form of JNDs and WFs.
Finally, discussion about possible implications of the results is presented in section 5 .

\section{STIFFNESS DISCRIMINATION}

We investigate stiffness discrimination abilities for virtual linear springs in the presence and absence of tactile feedback and visual feedback. Stiffness of spring can be defined as resistance of the spring to deformation by an applied force. It is one of the most studied properties of an object. Stiffness property can help us in the discrimination, identification, and manipulation of objects (Gurari et al., 2009). The stiffness value of a spring can be defined by the amount of deformation caused by a unit measure of an applied force (Li et al., 2015). Thus, the sensing stiffness of the spring requires the knowledge of both the amount of spring deformation (displacement) and associated forces. Our vision can provide us some information concerning the deformation of the spring; however, vision may not be able to provide us much information concerning the applied force (Paggetti et al., 2014).

A spring with rigid mass attached to it is selected over a deformable surface due to the complexity of tactile information from deformable surfaces. When a deformable compliant object comes in contact with the fingerpad, the mechanical variables of the contact interface at any instant of time are the net force constituting the applied force and the reactive force, area of contact, pressure distribution within the contact region, and displacement or deformation within the object and the fingerpad. Thus, an analysis of a deformable surface is not easy. In the case of rigid spring, unlike deformable surfaces the area of contact, average pressure distribution and skin fingerpad displacement are completely dependent on the net force of contact and are independent of the stiffness of the spring (Jang and Lee, 2014). Therefore, these parameters can be ignored during stiffness encoding.

Many studies have been done to quantify human stiffness discrimination abilities, using a variety of experimental methods (Roland and Ladegaard-Pedersen, 1977; Tan et al., 1995; Lecuyer et al., 2000). The study of discrimination of physical parameters such as stiffness can help us measure how well humans can discriminate these parameters (Koçak et al., 2011). In addition, the knowledge gained from the psychophysical studies performed after discrimination experiments can be used for the design of better haptic interfaces. During the design of haptic systems for virtual environment, it is important to consider the resolution of the haptic display to that of the human sensory systems (Tan et al., 1993; Wurdemann et al., 2013; Papetti et al., 2017).

Tactile information alone may not be sufficient in discrimination of stiffness. The sufficiency of tactile information for discrimination of stiffness for different objects can be explained by the mechanics of the finger-pad contact and its effect on the tactile information encoded (Srinivasan and LaMotte, 1995). For compliant object with rigid surfaces tactile information is not sufficient during stiffness discrimination, however, visual feedback can replace the proprioceptive sense to some extent. The effect of visual information on stiffness discrimination was explored in the studies (Srinivasan et al., 1996; Wu et al., 1999). For deformable surfaces, the spatial pressure distribution within 
the contact region depends both on the force applied and object compliance.

Different stiffness exploratory procedures have been studied (Freyberger and Färber, 2006). The stiffness of an object can be perceived by tapping or pressing. A common method to assess stiffness of an object is to squeeze the object between the index and thumb finger-pads or press with the index finger (Freyberger and Färber, 2006). The normal force applied on the object causes corresponding deformation of both the object and the fingerpad. Some studies show that discrimination was significantly better when tapping than pressing the object (LaMotte, 2000). This can be due to presence of tactile cues generated by the rapid increase of force rate during tapping. During tapping, the harder the object, the greater the amount of net force generated by the magnitude and rate of compressional forces against the object. Subjects could discriminate differences in stiffness by tactile cues in the absence of kinesthetic when the object is tapped at approximately the same velocity. The difference in applied velocity doesn't affect the discrimination while during pressing a small variation in applied velocity affects the discrimination specially during the absence of kinesthetic cues (LaMotte, 2000).

Many studies explore different techniques to display stiffness using haptic devices. Nonetheless to the best of our knowledge, none of these experiments use modulation of vibrotactile cutaneous cues to display stiffness for virtual interaction. Human can perceive stiffness changes as change of vibration force strength. Modulation of vibrotactile cues create range of vibration forces which can be used for discrimination of stiffness, pressure, weight, and Force. Therefore, in our context stiffness discrimination is implemented as discrimination of different vibration intensities while tapping a virtual spring. Tapping is selected over pressing due to the reasons mentioned in the previous paragraph. In addition, during tapping vibration is displayed for very short amount of time while pressing needs a continuous vibration while the finger is in contact with virtual object, which results in destructing effects of vibration and bias during tests.

\section{METHODS}

We built a single and multiple finger vibrotactile device (shown in Figure 1) which takes touch feedback signal from a VR haptic experimental setup developed in Unity 3D environment (https://unity3d.com/). The application is used to perform a two alternative forced choice psychological experiments to characterize the discrimination of stiffness using the vibrotactile device.

\subsection{Vibrotactile Haptic System}

The haptic setup is composed of ERM vibro-tactile actuators (Precison Micro-drives, 310-113) and haptic controllers (Adafruit, DRV2605) that drive them. The electronic controller consists of serial Bluetooth module (Bluetooth RF Transceiver HC-06) and a microcontroller (Adafruit Flora) that handles the logic and communication with the PC. The overall architecture of the haptic system hardware is shown in Figure 2.

ERM actuators are miniature DC motors with an offset mass attached to their shaft. The rotation of the mass causes a displacement of the motor due to asymmetry which results in vibration perpendicular to its rotation axis. Vibration intensity is controlled varying the voltage through PWM command between 0 and $5 \mathrm{~V}$. Due to the voltage constant of such actuators, at the maximum voltage command the motors, with an eccentric mass $(0.16 \mathrm{~g})$ mounted on its shaft. The frequencies reach an estimated value of about $500 \mathrm{~Hz}$. The frequency of vibration is $\omega / 60$, where $\omega$ is the rotational speed of the motor in rev per minute (rpm). This speed is proportional to the potential difference across the terminal of the motor. One limitation of vibration motors is that they cannot be driven to change its frequency and amplitude independently. The relationship between the vibration amplitude and frequency vs the input voltage is shown in Figure 3. As shown in this figure, the variation of voltage changes the frequency and amplitude of ERM actuators in a coupled fashion.

To this end, microcontrollers typically generate a PWM signal. This type of digital signal can change the average voltage by varying the burst width (or duty) of a periodic signal. The modulated signal is then used to feed a motor driver which is able to provide enough current for the motor winding. The modulated vibration strength can, therefore, be used in encoding haptic stimulus such as pressure or stiffness.

In Haptic applications, there is a delay between the detection of a user movement and the production of the haptic feedback. One of the drawbacks of using ERM actuators is their
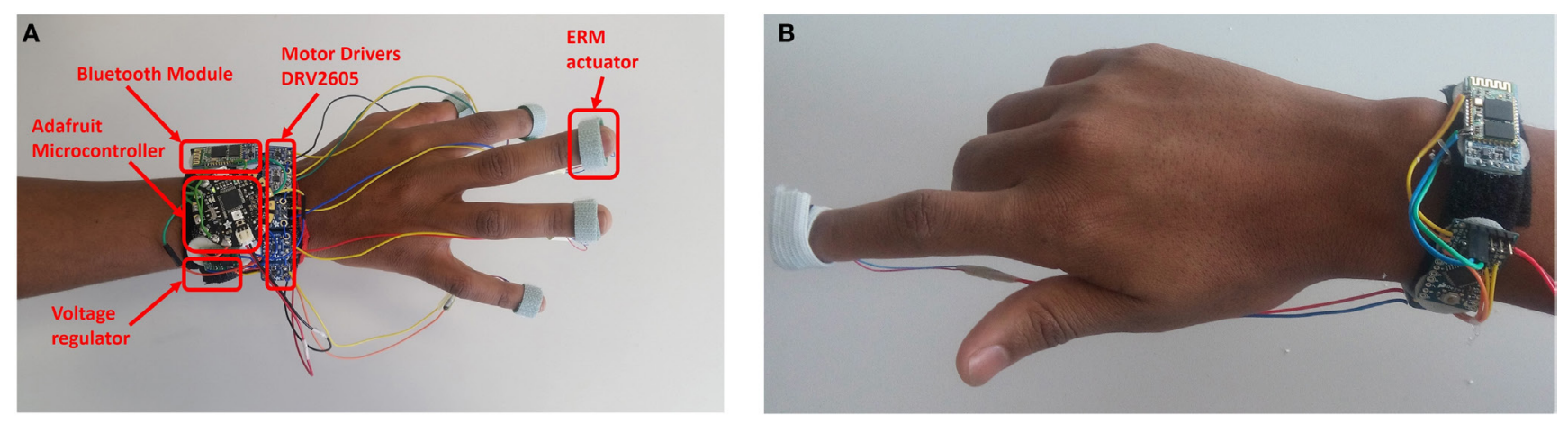

FIGURE 1 | Wearable vibrotactile haptics setup: (A) five finger haptic setup and (B) single finger haptic setup. 


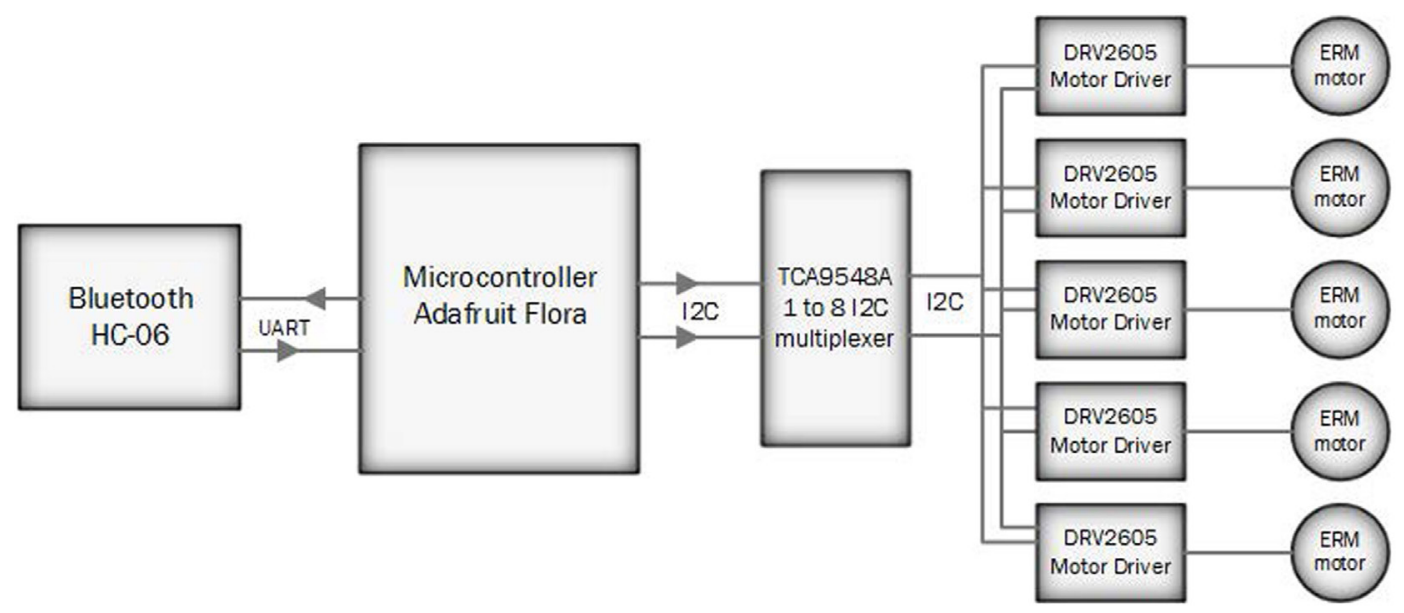

FIGURE 2 | Wireless Vibrotactile haptics system architecture for five fingers stimulation.

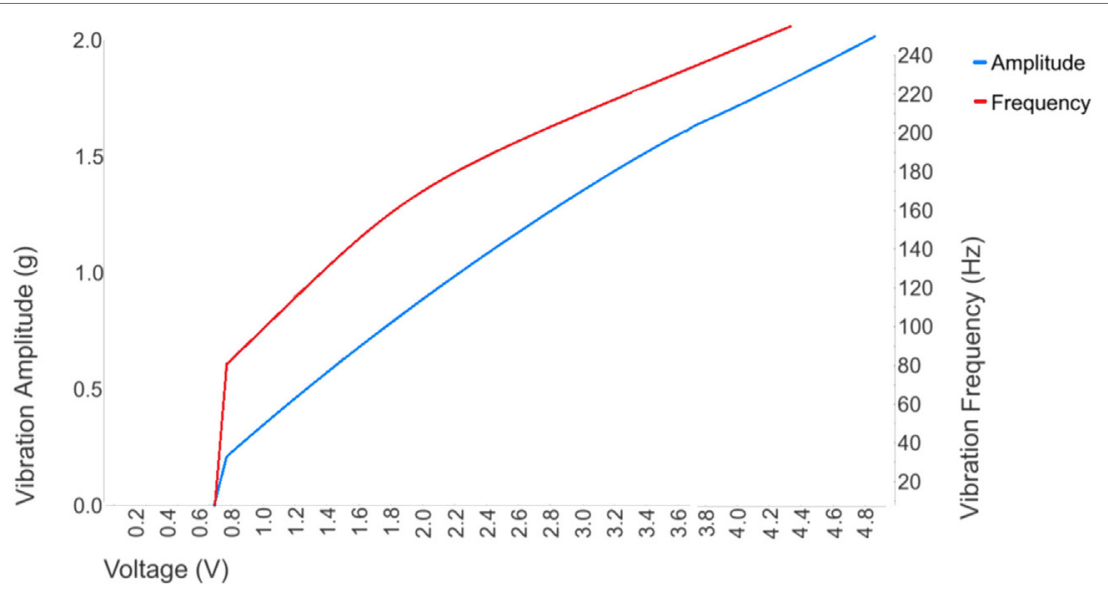

FIGURE 3 | Amplitude and Frequency relationship with Input Voltage.

relatively high latency to achieve the desirable output level at a given time. This latency can be caused by two factors: the time it takes for the motor to accelerate and the time taken to decelerate. A typical disk type vibrator takes approximately $200 \mathrm{~ms}$ to start and $250 \mathrm{~ms}$ to stop completely. In order to reduce these limitations, a dedicated controller DRV2605 is used. The DRV2605 is a very little motor driver from TI. It is designed to specially control haptic motors. The DRV2605 has an automatic overdrive and braking feedback methods, which creates more reliable motor control. To reduce the start time, the DRV2605 has an overdrive feature that applies higher loop gain to transient response of the actuator behavior. This method reduces the rise time to $40-60 \mathrm{~ms}$ with an overdrive voltage of $3 \mathrm{~V}$. To reduce the brake time of the actuator, the DRV2605 provide a means to increase the gain ratio of the closed loop system (see Texas instruments, DRV2605 datasheet, p. 15, Table 1).

The ERM motors could also be controlled with MOSFET transistor circuits and other motor drivers using generated PWM signal from a microcontroller. The PWM modulated signal is feed to the driver which is able to provide enough current for the motor. However, DRV2605 has the ability to drive the motors with advanced and already defined waveform patterns to convey information to the user. Short and long click, ramp, buzz, and hum effects can be triggered. It is also possible to trigger multiple predefined haptic waveforms consequently. The DRV2605 chip is controlled over I2C, but since all the DRV2605 drivers comes with the same I2C address, it makes it impossible to have more than one device on the same SDA/SCL pins. This problem, however, can be easily solved using TCA9548A 1-to-8 multiplexer. Even though the multiplexer itself is on $\mathrm{I} 2 \mathrm{C}$ address $0 \times 70$, it can be adjusted from $0 \times 70$ to $0 \times 77$. This chip enables to controller up to 8 same address DRV2605 driver connected to the same I2C port. The multiplexer puts the commands from the microcontroller to the selected motor driver. The microcontroller has a function of choosing the correct motor driver and desired waveform type according to the finger type used to interact in virtual environment. 


\subsection{Virtual Experimental Setup}

The stimulation of human senses in VR has been limited mainly to the visual and auditory channels. Our system couples a simple game (shown in Figure 4) created in Unity 5.3 game engine with built-in VR support and a wearable haptic device. Unity allows the integration of Oculus and Leap motion for creation of highly immersive virtual environment as shown in Figure 5. For the experimental setup, we choose virtual springs with prescribed spring strength values. A virtual spring is chosen for stiffness discrimination experiment because of the simplicity of the model of stiffness, relevant previous works on manual discrimination of stiffness, and the facts that springs are classical fundamental elements of virtual computer models. The perception of the stiffness of the spring involves a multi-modal combination of force and displacement. The force information is derived from the reactive force from ones interaction with the spring. Therefore, the displacement of the spring and reactive force encoded on haptic devices allow the user to discriminate the stiffness of the virtual springs.

The method implemented to detect the contact between a virtual hand and the virtual springs utilizes Unity collision detection system. The virtual collision is synchronized with the haptic force feedback rendering. Collision information, including spring displacement and spring constant (spring strength), are used to calculate the spring force at any instant of collision time.

A mass spring model shown in Figure $\mathbf{6}$ is considered for virtual objects force rendering. The spring forces are used to programmatically control and create a range of vibration effects by the haptic device. The interaction force is represented according to Hooke's law:

$$
F=k * x
$$

where $k$ represents the stiffness parameter and $x$ is the displacement of the spring during compression. According to the above equation, given that stiffness is defined as ratio of force and displacement, a psychophysical experiment characterize the perception of stiffness from visual (displacement) and haptic (force) variables.

This equation is used to render the interaction force displayed through the haptic devices. Spring force rendering with the haptic device is processed with a thread that iterates at $500 \mathrm{~Hz}$ which is also the maximum frequency achieved by the ERM motors. This ensures that vibration forces generated or transition between vibration forces are smooth.

\subsection{Psychophysics Experiments}

Psychophysics is one of the disciplines contributing to the design and research of more effective haptic hardware and software solution by understanding our perception mechanisms (Lecuyer et al., 2000). One of the common psychometric method to study the effectiveness of haptic device in exploring stiffness of an object is the Two Alternative Forced Choice (2AFC) method. This method uses a method of varying the levels of stiffness to get the Just Noticable difference (JND). JND and Weber Fraction (WF) are used to make a quantitative analysis of the discrimination behaviors. The JND is the measure of the minimal difference

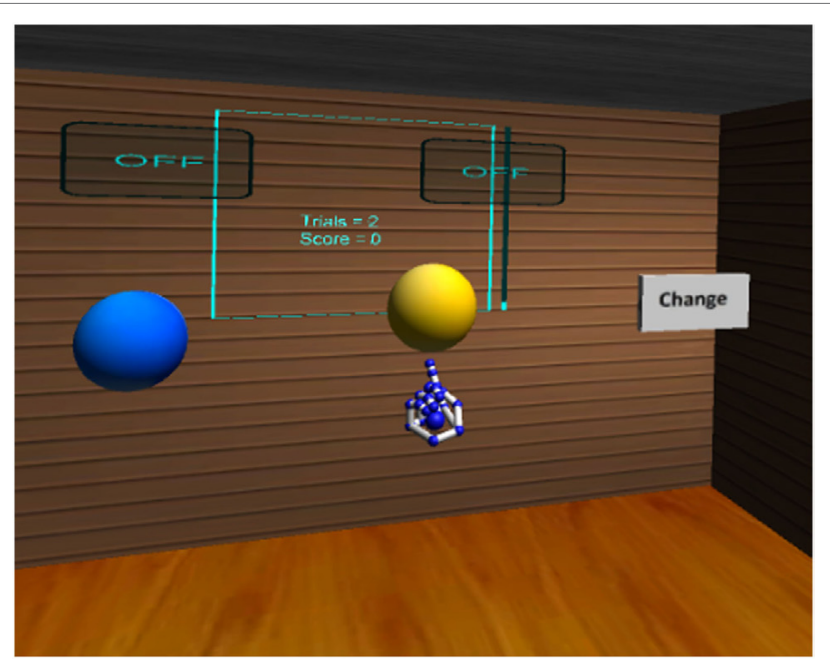

FIGURE 4 | Virtual experimental setup screen made in Unity 3D.

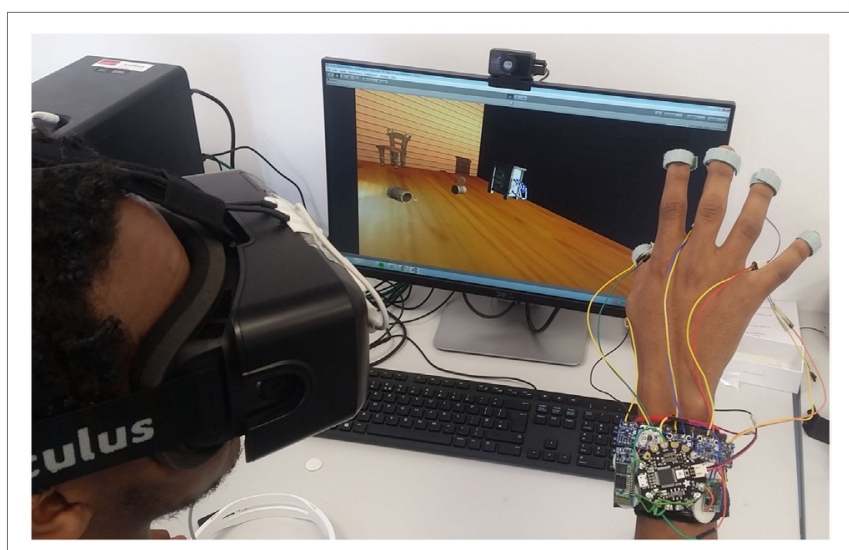

FIGURE 5 | Virtual reality setup with Oculus Rift and Leap Motion.

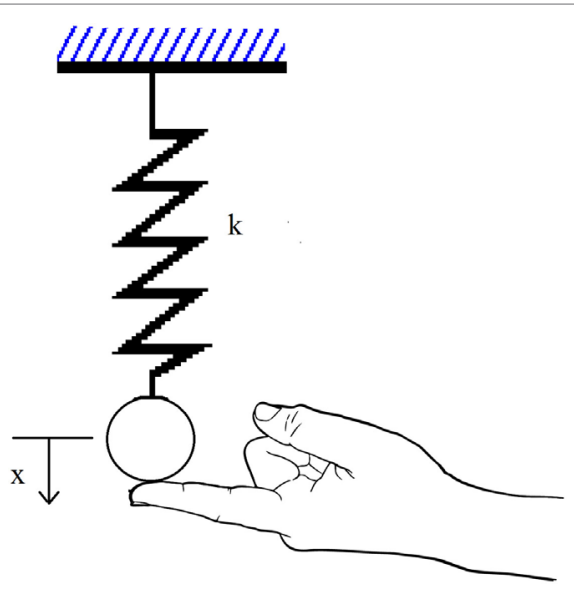

FIGURE 6 | Virtual spring mass model. 
between two intensities of the stimulus that can be discriminated by our perceptual experience (Koçak et al., 2011; Genecov et al., 2014). WFs are used as a measure of one's ability to discriminate the stiffness between springs.

Three feedback modalities are tested:

- Visual Only: the subjects distinguish the stiffness by tapping the spring in the absence of tactile feedback. The displacement of the virtual spring was visually observed on a computer screen.

- Tactile Only: the subject is delivered with tactile feedback only. Without looking at the spring visual characteristics, the participant is supposed to make the decision based on vibrotactile feedback on his index finger.

- Combination of both visual and tactile feedback.

In these experiments, ten participants, five female and five male within the ages of 21 and 33 years have participated. All of them have no known perception disorder and eight of them are right handed while two are left handed. To start the experiment, participants signed the consent form, then they were introduced to the experiment within 4- to 5-min practice session by randomly playing with the virtual experimental setup. Then, all three feedback modalities and the subjects were presented with two springs: a standard (reference) spring with threshold spring strength value and four comparison springs with prescribed stiffness values.

A pair of springs from five virtual spring models, $S_{1}, S_{2}, \ldots$, $S_{5}$, enabling five different stiffness values are displayed on screen. The stiffness values are assigned as Unity spring strength constant values of $5,20,35,50$, and 65 with $k s_{3}=35$ being the reference stiffness value. These five stiffness values correspond to 20, 40, 60,80 , and $100 \%$ PWM duty cycles, respectively. The springs were presented in random order selected from a set of five spring strength (stiffness) values. The user can explore the springs for unlimited amount of time. The users decision about the stiffness of the spring depends on the vibration strength felt at the fingertip. For 2AFC test, the user compares vibration strength while tapping the two springs and decides which spring feels stiffer which means the user decides if the comparison spring is stiffer or softer than the reference spring. After decision made about the stiffness of the springs, buttons corresponding to both springs are available to choose. The subject can also switch between springs as many times as desired. Number of total trials and number of correct trials (scores) are also displayed on virtual screen. In order to switch to another spring, virtual buttons are also available. Pressing this button marked the completion of a trial. Following each trial, corresponding data, such as trial number, compared spring stiffness values, and subjects choice, are automatically collected by the program and saved to a file.

Each of the five stiffness values appears randomly once in 5 trials. Ten sets of these 5 trials were repeated with another random order. Therefore, a participant makes 50 trials for each feedback modality. To avoid biases due to fatigue and reduce finger insensitivity due to long vibrations, the subjects were forced to take a break after completing one feedback modality. All ten participants completed the experiment for the three feedback modalities in the order of visual only, tactile only, and combination. In order to avoid bias which can be created by visual learning of the subjects, stiffness values, colors of virtual springs, and display position of springs are assigned randomly during run time. This also helps us to reduce a significant bias caused by the order of the test modalities. The average percentage of correct response for different spring combinations in different modalities is collected as shown in Figure 4.

\subsubsection{Psychometric Curve}

For all experiments, the proportion of time the user correctly identified the comparison stiffness as higher than the standard stiffness of the springs is plotted against the five stiffness values. A sigmoid function of the form

$$
\frac{1}{1+e^{-\left(\frac{x-\alpha}{\beta}\right)}}
$$

is used to fit the plot points with curve fitting functions. The psychometric curve fit shown in Figure 7 is made using LabVIEW curve fitting functions.

The $\alpha$ and $\beta$ values for the plots of average values from all three modalities are as shown in Table 1.

From each psychometric curve, we obtain Point of Subjective Equality (PSE), the point where the subject perceives the two stiffness values to be equal, or the stiffness value corresponding to $50 \%$ of correct responses. We also found the lower and upper Just Noticeable Difference (JND) from the 25 and $75 \%$ of correct responses, respectively. Then the JND can be computed by averaging the upper and lower JNDs as in the following equation:

$$
\begin{aligned}
& J N D_{\text {lower }}=P S E-k_{25 \%} \\
& J N D_{\text {upper }}=k_{75 \%}-P S E .
\end{aligned}
$$

The WF is also computed as the ratio of JNDs and PSE:

$$
\begin{gathered}
J N D=\frac{J N D_{\text {upper }}+J N D_{\text {lower }}}{2} \\
W F=\frac{J N D}{P S E} .
\end{gathered}
$$

\section{RESULTS}

The results of the psychophysical experiment are summarized in Table 2 in the form of WF values. The mean JNDs $( \pm S E)$ are $1.115( \pm 0.55)$ for visual only, $0.694( \pm 0.17)$ for tactile only, and $0.709( \pm 0.27)$ for combination. A one-way ANOVA test has been conducted using SPSS statistics to see if there is a statistically significant difference between the means of JND Values found in the three modality tests. The JNDs for all 10 subjects, reported in Table 3 is taken as sample data against the independent samples of three modalities $(k=3)$. A statistical analysis with one way ANOVA on JNDs reveal a significant difference between the means of three types of feedback $[\mathrm{F}(2,27)=4.79$, $\mathrm{p}=0.0165$.

The mean WF $( \pm S E)$ for stiffness perception is $0.393( \pm 0.204)$ for visual only feedback, 0.238 ( \pm 0.061) for tactile only feedback, 


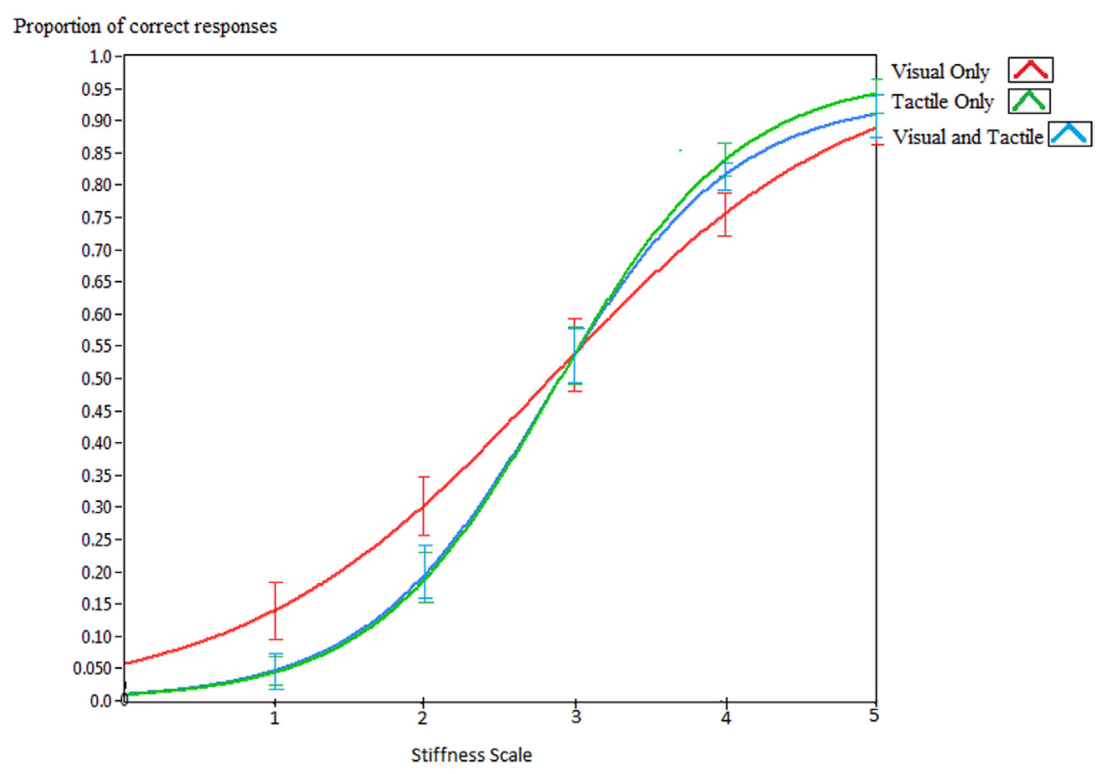

FIGURE 7 | Average Psychometric curve for all three feedback modalities: Visual only, Tactile only, Visual and Tactile.

TABLE 1 $\mid \alpha$ and $\beta$ variables for each psychometric curve fit.

\begin{tabular}{lcc}
\hline Feedback type & $\boldsymbol{\alpha}$ & $\boldsymbol{\beta}$ \\
\hline Visual & 2.8198 & 1.0087 \\
Tactile & 2.8668 & 0.6102 \\
V and T & 2.8282 & 0.5899 \\
\hline
\end{tabular}

TABLE 2 | Weber Fractions.

\begin{tabular}{cccc}
\hline Subjects & Visual only & Tactile only & V and T \\
\hline 1 & 0.316 & 0.217 & 0.232 \\
2 & 0.503 & 0.287 & $\mathbf{0 . 1 8 2}$ \\
3 & 0.291 & 0.357 & 0.366 \\
4 & $\mathbf{0 . 1 7 1}$ & $\mathbf{0 . 1 5 8}$ & $\mathbf{0 . 1 6 3}$ \\
5 & 0.474 & 0.302 & 0.204 \\
6 & 0.482 & $\mathbf{0 . 1 9 5}$ & 0.486 \\
7 & $\mathbf{0 . 1 9 4}$ & 0.212 & $\mathbf{0 . 1 9 6}$ \\
8 & 0.876 & 0.207 & 0.219 \\
9 & 0.298 & $\mathbf{0 . 1 8 0}$ & 0.220 \\
10 & 0.467 & 0.244 & $\mathbf{0 . 1 8 6}$ \\
Mean & 0.393 & 0.238 & 0.244 \\
\hline
\end{tabular}

Bold letters are used just to indicate subject which shows better performance on each test modalities.

and $0.244( \pm 0.099)$ for combination of visual and tactile feedback. A one-way ANOVA on WF values is also calculated with WF values of all 10 subjects reported in Table 2 as sample data against the independent samples of three test modalities. The statistical results indicated there is a significant difference between the means of the three conditions $[\mathrm{F}(2,27)=4.84, \mathrm{p}=0.0159]$. A Tukey HSD test also done on the same sample data to study which of these WF values statistically differ from each other. The HSD test result shows the mean WF were not significantly different between tactile only and combination tests.
TABLE 3 | PSE and GND values.

\begin{tabular}{|c|c|c|c|c|c|c|}
\hline \multirow{2}{*}{$\frac{\text { Mode }}{\text { Subjects }}$} & \multicolumn{2}{|c|}{ Visual } & \multicolumn{2}{|c|}{ Tactile } & \multicolumn{2}{|c|}{$V$ and $T$} \\
\hline & PSE & JND & PSE & JND & PSE & JND \\
\hline 1 & 2.720 & 0.861 & 2.749 & 0.597 & 2.925 & 0.680 \\
\hline 2 & 2.886 & 1.453 & 2.837 & 0.817 & 3.052 & 0.557 \\
\hline 3 & 2.671 & 0.778 & 2.700 & 0.965 & 2.749 & 1.007 \\
\hline 4 & 2.563 & 0.440 & 2.866 & 0.453 & 2.778 & 0.455 \\
\hline 5 & 2.915 & 1.384 & 3.070 & 0.929 & 3.023 & 0.616 \\
\hline 6 & 3.258 & 1.472 & 3.180 & 0.621 & 2.857 & 1.389 \\
\hline 7 & 3.062 & 0.594 & 2.739 & 0.582 & 2.808 & 0.552 \\
\hline 8 & 2.632 & 2.307 & 3.043 & 0.631 & 3.082 & 0.675 \\
\hline 9 & 2.700 & 0.807 & 2.906 & 0.523 & 2.974 & 0.655 \\
\hline 10 & 3.06 & 1.43 & 2.89 & 0.705 & 2.994 & 0.557 \\
\hline Mean & 2.837 & 1.115 & 2.906 & 0.694 & 2.906 & 0.709 \\
\hline
\end{tabular}

A one-way ANOVA has also been used to show the difference of the mean for the proportion of correct responses among stiffness levels. To this end, proportion of correct responses of visual, tactile, and combination tests are taken as samples against the 5 stiffness levels as independent samples $(k=5)$. The test shows a significant difference $[\mathrm{F}(4,45)=123.3, \mathrm{p}<0.0001]$ for visual, $[\mathrm{F}(4,45)=323.2, \mathrm{p}<0.0001]$ for tactile, and $[\mathrm{F}(4,45)=271.9$, $\mathrm{p}<0.001]$ for combination of the visual and tactile feedback. A Tukey HSD test on the same samples shows that there is no significant difference on average responses for comparisons of stiffness values $k_{s 1}=5$ and $K_{s 2}=20$ for all feedback modalities.

\subsection{Post Study Survey}

After finishing the experiment, the subjects were provided with a questionnaire for qualitative analysis of the experiment. The survey is done to study the difficulty of the task and the realism of the haptic perception experienced. From survey collected at the end of the experiment, the user experience with haptic devices 


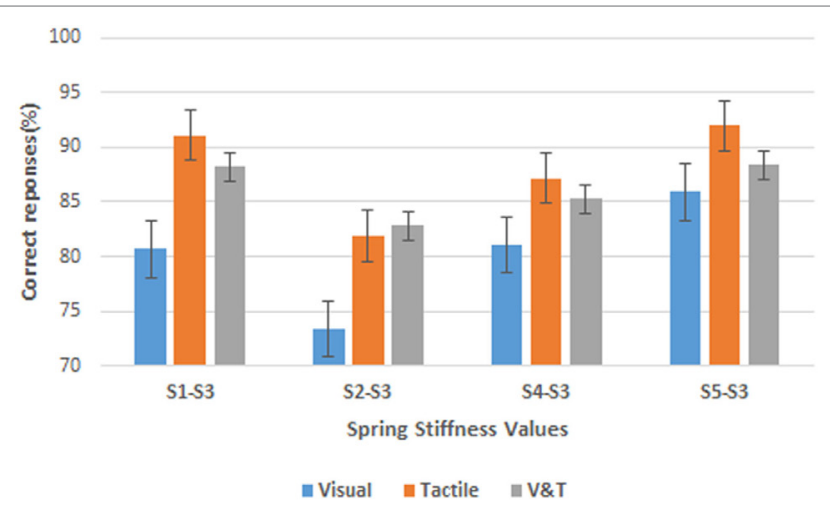

FIGURE 8 | Percentage of correct responses during comparison of each spring with the standard spring for all modalities.

ranged from 1 to 5 , with an average of 0.85 . The users also rate the difficulty of the task from 1 (not difficult) to 5 (very difficult) with an average of 1.6. Furthermore, all subjects found that the model of the spring was visually realistic. However, subjects rate the haptic sensation realism with an average of 3.8 on scale 1 (not realistic at all) to 5 (highly realistic).

\section{DISCUSSION}

The psychometric experiments in general shows that the vibrotactile device can enhance the perception of stiffness than visual only feedback. The result shows the average JND of $22.3 \%$ for visual only feedback is reduced to $14.7 \%$ during combination of both visual and tactile feedback. The result from previous experiments found $8-22 \%$ JNDs (Tan et al., 1992). The herein result seems consistent with previous studies concerning discrimination of stiffness (Jones and Hunter, 1992; Tan et al., 1992; Jang and Lee, 2014).

Figure 8 and Table 3 show that tactile only and combination of visual and tactile feedback equally helps in discriminating stiffness values less than the standard spring stiffness and also the PSE values is the equal. For stiffness values greater than the standard value tactile, only feedback shows better performance than the combination of visual and tactile feedback. The interference of one perceptual stimulus with one another causing a decrease in perceptual effectiveness, or perceptual masking can also affect the users performance. The other implication could also be cognitive capabilities with processing multiple stimuli simultaneously. Previous experiments observed that vision could also mislead someone during stiffness discrimination tasks. Inappropriate

\section{REFERENCES}

Carr, K., and England, R. (1995). Simulated and Virtual Realities: Elements of Perception. CRC Press.

Connelly, L., Jia, Y., Toro, M. L., Stoykov, M. E., Kenyon, R. V., and Kamper, D. G. (2010). A pneumatic glove and immersive virtual reality environment for hand rehabilitative training after stroke. IEEE Trans. Neural Syst. Rehabil. Eng. 18, 551-559. doi:10.1109/TNSRE.2010.2047588 vision feedback can also totally invert the stiffness perception and result in biases of experiment (Hachisu et al., 2011). Such kind of mistake by illusion is made by the brain, not our senses.

As shown in Table 2, two participants show good capability on visual only stiffness discrimination, by contrast, most of the participants shows better tactile stiffness perception. This result can also be due to different sensory weighting of participants for visual and tactile senses. The mean JNDs and WFs are within the range of results from measured human perception of stiffness from other devices. For example, the WFs obtained in these experiments are smaller to stiffness discrimination tasks reported in Gurari et al. (2009). Shon and McMains (2004) compute the WF 0.67 of the base stimulus for stiffness discrimination.

\section{CONCLUSION}

Design of an effective haptic system for virtual interaction requires sufficient understanding of human perceptual systems and haptic exploration strategies. We conducted experiments to study the effectiveness of vibrotactile cutaneous feedback in stiffness discrimination in the absence of kinesthetic feedback. However, visual feedback is used to compensate the kinesthetic feedback. Perceptual experiments for stiffness discrimination have been done and data from all subjects fit to a psychometric functions. The Comparison of WF for visual and tactile feedback modalities and WF comparisons with previous experiments average WF indicates even though low cost and very portable, our device can be effectively used for application which needs virtual stiffness discrimination such as surgical simulators and virtual prototyping (Howard and Szewczyk, 2016).

\section{ETHICS STATEMENT}

This study was approved by the Research Ethics Sub-Committee of the Faculty of Science in Liverpool Hope University. All subjects read and sign the committee approved research consent form. Subjects have been informed about the procedures and expectations and they were also be informed that they can withdraw from the experiment if the Oculus Rift causes them discomfort (motion sickness) during the study.

\section{AUTHOR CONTRIBUTIONS}

AM: system development, experimental protocol development, data acquisition and processing, literature review, experimental setup, manuscript writing, and manuscript review. ES: experimental design and manuscript review. DR: literature review and manuscript review. AN: literature review and manuscript review. 
Genecov, A. M., Stanley, A. A., and Okamura, A. M. (2014). "Perception of a haptic jamming display: just noticeable differences in stiffness and geometry," in 2014 IEEE Haptics Symposium (HAPTICS) (IEEE), 333-338.

Gurari, N., Kuchenbecker, K. J., and Okamura, A. M. (2009). "Stiffness discrimination with visual and proprioceptive cues," in EuroHaptics Conference, 2009 and Symposium on Haptic Interfaces for Virtual Environment and Teleoperator Systems. World Haptics 2009. Third Joint (IEEE), 121-126.

Hachisu, T., Cirio, G., Marchal, M., Lécuyer, A., and Kajimoto, H. (2011). "Pseudohaptic feedback augmented with visual and tactile vibrations," in 2011 IEEE International Symposium on VR Innovation (ISVRI) (IEEE), 327-328.

Howard, T., and Szewczyk, J. (2016). Improving precision in navigating laparoscopic surgery instruments toward a planar target using haptic and visual feedback. Front. Rob. AI 3:37. doi:10.3389/frobt.2016.00037

Jang, I., and Lee, D. (2014). "On utilizing pseudo-haptics for cutaneous fingertip haptic device," in 2014 IEEE Haptics Symposium (HAPTICS) (IEEE), 635-639.

Jones, L. A., and Hunter, I. W. (1992). Human operator perception of mechanical variables and their effects on tracking performance. ASME Dyn. Syst. Control. Div. Publ. 1992, 49-53.

Kaczmarek, K. A., Webster, J. G., Bach-y Rita, P., and Tompkins, W. J. (1991). Electrotactile and vibrotactile displays for sensory substitution systems. IEEE Trans. Biomed. Eng. 38, 1-16. doi:10.1109/10.68204

Koçak, U., Palmerius, K. L., Forsell, C., Ynnerman, A., and Cooper, M. (2011). "Analysis of the jnd of stiffness in three modes of comparison," in International Workshop on Haptic and Audio Interaction Design (Springer), 22-31.

LaMotte, R. H. (2000). Softness discrimination with a tool. J. Neurophysiol. 83, 1777-1786.

Lecuyer, A., Coquillart, S., Kheddar, A., Richard, P., and Coiffet, P. (2000). "Pseudohaptic feedback: can isometric input devices simulate force feedback?" in Proceedings IEEE Virtual Reality, 2000 (IEEE), 83-90.

Li, M., Konstantinova, J., Secco, E. L., Jiang, A., Liu, H., Nanayakkara, T., et al. (2015). Using visual cues to enhance haptic feedback for palpation on virtual model of soft tissue. Med. Biol. Eng. Comput. 53, 1177-1186. doi:10.1007/ s11517-015-1309-4

Li, M., Luo, S., Nanayakkara, T., Seneviratne, L. D., Dasgupta, P., and Althoefer, K. (2014). Multi-fingered haptic palpation using pneumatic feedback actuators. Sens. Actuators A Phys. 218, 132-141. doi:10.1016/j.sna.2014.08.003

Lieberman, J., and Breazeal, C. (2007). Tikl: development of a wearable vibrotactile feedback suit for improved human motor learning. IEEE Trans. Robot. 23, 919-926. doi:10.1109/TRO.2007.907481

Minamizawa, K., Prattichizzo, D., and Tachi, S. (2010). "Simplified design of haptic display by extending one-point kinesthetic feedback to multipoint tactile feedback," in 2010 IEEE Haptics Symposium (IEEE), 257-260.

Pabon, S., Sotgiu, E., Leonardi, R., Brancolini, C., Portillo-Rodriguez, O., Frisoli, A., et al. (2007). "A data-glove with vibro-tactile stimulators for virtual social interaction and rehabilitation," in 10th Annual Intl Workshop on Presence.

Pacchierotti, C., Chinello, F., Malvezzi, M., Meli, L., and Prattichizzo, D. (2012). "Two finger grasping simulation with cutaneous and kinesthetic force feedback," in International Conference on Human Haptic Sensing and Touch Enabled Computer Applications (Springer), 373-382.

Paggetti, G., Cizmeci, B., Dillioglugil, C., and Steinbach, E. (2014). "On the discrimination of stiffness during pressing and pinching of virtual springs," in
2014 IEEE International Symposium on Haptic, Audio and Visual Environments and Games (HAVE) (IEEE), 94-99.

Papetti, S., Järveläinen, H., Giordano, B. L., Schiesser, S., and Fröhlich, M. (2017). Vibrotactile sensitivity in active touch: effect of pressing force. IEEE Trans. Haptics 10, 113-122. doi:10.1109/TOH.2016.2582485

Roland, P., and Ladegaard-Pedersen, H. (1977). A quantitative analysis of sensations of tension and of kinaesthesia in man. Brain 100, 671-692. doi:10.1093/ brain/100.4.671

Rose, J. E., and Mountcastle, V. B. (1959). Touch and kinesthesis. Handb. Physiol. 1, 387-429.

Shon, Y., and McMains, S. (2004). Evaluation of drawing on 3D surfaces with haptics. IEEE Comput. Graph. Appl. 24, 40-50. doi:10.1109/MCG.2004.49

Srinivasan, M. A., Beauregard, G. L., and Brock, D. L. (1996). "The impact of visual information on the haptic perception of stiffness in virtual environments," in ASME Winter Annual Meeting, Vol. 58, 555-559.

Srinivasan, M. A., and LaMotte, R. H. (1995). Tactual discrimination of softness. J. Neurophysiol. 73, 88-101.

Tan, H. Z., Durlach, N. I., Beauregard, G. L., and Srinivasan, M. A. (1995). Manual discrimination of compliance using active pinch grasp: the roles of force and work cues. Percept. Psychophys. 57, 495-510. doi:10.3758/BF03213075

Tan, H. Z., Durlach, N. I., Shao, Y., and Wei, M. (1993). Manual resolution of compliance when work and force cues are minimized. ASME Dyn. Syst. Control. Div. Publ. 1993, 99-104.

Tan, H. Z., Pang, X. D., and Durlach, N. I. (1992). Manual resolution of length, force, and compliance. Adv. Rob. 42, 13-18.

Van Erp, J. B. (2002). Guidelines for the use of vibro-tactile displays in human computer interaction. Proc. Eurohaptics 2002, 18-22.

Vicentini, M., and Botturi, D. (2010). Perceptual Issues Improve Haptic Systems Performance. INTECH Open Access Publisher.

Wu, W.-C., Basdogan, C., and Srinivasan, M. A. (1999). Visual, haptic, and bimodal perception of size and stiffness in virtual environments. ASME Dyn. Syst. Control. Div. Publ. 67, 19-26.

Wurdemann, H. A., Secco, E. L., Nanayakkara, T., Althoefer, K., Lis, K., Mucha, L., et al. (2013). "Mapping tactile information of a soft manipulator to a haptic sleeve in rmis," in 3rd Joint Workshop on New Technologies for Computer/Robot Assisted Surgery (CRAS 2013).

Yang, C., Zhang, J., Chen, Y., Dong, Y., and Zhang, Y. (2008). A review of exoskeleton-type systems and their key technologies. Proc. Inst. Mech. Eng. C 222, 1599-1612.

Conflict of Interest Statement: The authors declare that the research was conducted in the absence of any commercial or financial relationships that could be construed as a potential conflict of interest.

Copyright (c) 2017 Maereg, Nagar, Reid and Secco. This is an open-access article distributed under the terms of the Creative Commons Attribution License (CC BY). The use, distribution or reproduction in other forums is permitted, provided the original author(s) or licensor are credited and that the original publication in this journal is cited, in accordance with accepted academic practice. No use, distribution or reproduction is permitted which does not comply with these terms. 